\title{
UNIFORM STABILIZATION OF THE WAVE EQUATION ON COMPACT SURFACES AND LOCALLY DISTRIBUTED DAMPING*
}

\author{
M. M. CAVALCANTI ${ }^{\dagger}$, V. N. DOMINGOS CAVALCANTI ${ }^{\ddagger}$, R. FUKUOKA ${ }^{\S}$, AND \\ J. A. SORIANO
}

Abstract. This paper is concerned with the study of the wave equation on compact surfaces and locally distributed damping, described by

$$
\left.u_{t t}-\Delta_{\mathcal{M}} u+a(x) g\left(u_{t}\right)=0 \quad \text { on } \mathcal{M} \times\right] 0, \infty[,
$$

where $\mathcal{M} \subset \mathbb{R}^{3}$ is a smooth (of class $C^{3}$ ) oriented embedded compact surface without boundary, such that $\mathcal{M}=\mathcal{M}_{0} \cup \mathcal{M}_{1}$, where

$$
\mathcal{M}_{1}:=\{x \in \mathcal{M} ; m(x) \cdot \nu(x)>0\}, \text { AND } \mathcal{M}_{0}=\mathcal{M} \backslash \mathcal{M}_{1} .
$$

Here, $m(x):=x-x^{0},\left(x^{0} \in \mathbb{R}^{3}\right.$ fixed $)$ and $\nu$ is the exterior unit normal vector field of $\mathcal{M}$.

For $i=1, \ldots, k$, assume that there exist open subsets $\mathcal{M}_{0 i} \subset \mathcal{M}_{0}$ of $\mathcal{M}$ with smooth boundary $\partial \mathcal{M}_{0 i}$ such that $\mathcal{M}_{0 i}$ are umbilical, or more generally, that the principal curvatures $k_{1}$ and $k_{2}$ satisfy $\left|k_{1}(x)-k_{2}(x)\right|<\varepsilon_{i}\left(\varepsilon_{i}\right.$ considered small enough) for all $x \in \mathcal{M}_{0 i}$. Moreover suppose that the mean curvature $H$ of each $\mathcal{M}_{0 i}$ is non-positive (i.e. $H \leq 0$ on $\mathcal{M}_{0 i}$ for every $\left.i=1, \ldots, k\right)$. If $a(x) \geq a_{0}>0$ on an open subset $\mathcal{M} * \subset \mathcal{M}$ that contains $\mathcal{M} \backslash \cup_{i=1}^{k} \mathcal{M}_{0 i}$ and if $g$ is a monotonic increasing function such that $k|s| \leq|g(s)| \leq K|s|$ for all $|s| \geq 1$, then uniform decay rates of the energy hold.

Key words. Wave equation, localized damping, surfaces in R3, umbilical surfaces.

AMS subject classifications. 35L05, 35L15, 53A05, 53A10

1. Introduction. Let $\mathcal{M}$ be a smooth (of class $C^{3}$ ) oriented embedded compact surface without boundary in $\mathbb{R}^{3}$ with $\mathcal{M}=\mathcal{M}_{0} \cup \mathcal{M}_{1}$, where

$$
\mathcal{M}_{1}:=\{x \in \mathcal{M} ; m(x) \cdot \nu(x)>0\} \quad, \text { AND } \mathcal{M}_{0}=\mathcal{M} \backslash \mathcal{M}_{1}
$$

Here, $m(x):=x-x^{0},\left(x^{0} \in \mathbb{R}^{3}\right.$ fixed $)$ and $\nu$ is the exterior unit normal vector field of $\mathcal{M}$.

We denote by $\nabla_{T}$ the tangential-gradient on $\mathcal{M}$, by $\Delta_{\mathcal{M}}$ the Laplace-Beltrami operator on $\mathcal{M}$. This paper is devoted to the study of the uniform stabilization of solutions of the following damped problem

$$
\left\{\begin{array}{lc}
u_{t t}-\Delta_{\mathcal{M}} u+a(x) g\left(u_{t}\right)=0 & \text { on } \mathcal{M} \times] 0, \infty[, \\
u(x, 0)=u^{0}(x), \quad u_{t}(x, 0)=u^{1}(x) & x \in \mathcal{M},
\end{array}\right.
$$

where $a(x) \geq a_{0}>0$ on an open proper subset $\mathcal{M}_{*}$ of $\mathcal{M}$ and in addition $g$ is a monotonic increasing function such that $k|s| \leq|g(s)| \leq K|s|$ for all $|s| \geq 1$.

Stability for the wave equation

$$
u_{t t}-\Delta u+f(u)+a(x) g\left(u_{t}\right)=0 \text { in } \Omega \times \mathbb{R}_{+},
$$

\footnotetext{
*Received July 14, 2008; accepted for publication July 16, 2008.

$\dagger$ †epartment of Mathematics, State University of Maringá, 87020-900, Maringá, PR, Brazil (mm cavalcanti@uem.br). Partially supported by the CNPq Grant 300631/2003-0.

¥Department of Mathematics, State University of Maringá, 87020-900, Maringá, PR, Brazil (vnd cavalcanti@uem.br). Partially supported by the CNPq Grant 304895/2003-2.

${ }^{\S}$ Department of Mathematics, State University of Maringá, 87020-900, Maringá, PR, Brazil (rfukuoka@uem.br).

IDepartment of Mathematics, State University of Maringá, 87020-900, Maringá, PR, Brazil (jas palomino@uem.br). Partially supported by the CNPq Grant 301352/2003-8.
} 
where $\Omega$ is a bounded domain in $\mathbb{R}^{n}$, has been studied for long time by many authors. When the feedback term depends on the velocity in a linear way Zuazua [ZUA] proved that the energy related to the above equation decays exponentially if the damping region contains a neighborhood of the boundary $\partial \Omega$ of $\Omega$ or, at least, contains a neighborhood $\omega$ of the particular part given by $\left\{x \in \partial \Omega:\left(x-x_{0}\right) \cdot \nu(x) \geq 0\right\}$. In the same direction, but when $f=0$, it is important to mention the work due to Rauch and Taylor [Ra-Ta] and, subsequently, the results of Bardos, Lebeau and Rauch [BAR], based on microlocal analysis, that ensures a necessary and sufficient condition to obtain exponential decay, namely, the damping region satisfies the well known geometric control condition. The classical example of an open subset $\omega$ verifying this condition is when $\omega$ is a neighborhood of the boundary. Later, again considering $f=0$, Nakao [Na1, Na2] extended the results of Zuazua [ZUA] treating first the case of a linear degenerate equation, and then the case of a nonlinear dissipation $\rho\left(x, u_{t}\right)$ (here, again, $f=0$ was considered) assuming, as usually, that the function $\rho$ has a polynomial growth near the origin. Martinez [Mar] improved the previous results mentioned above in what concerns the linear wave equation subject to a nonlinear dissipation $\rho\left(x, u_{t}\right)$, avoiding the polynomial growth of the function $\rho(x, s)$ in zero. His proof is based on the piecewise multiplier technique developed by Liu [Liu] combined with nonlinear integral inequalities to show that the energy of the system decays to zero with a precise decay rate estimate if the damping region satisfies some geometrical conditions. More recently, and still considering $f=0$, Alabau-Boussouira [ALA] extended the results due to Martinez [Mar] by showing optimal decay rates of energy. In addition, we would like to mention the most recent work in this direction due to D. Toundykov [Tou] which presents optimal decay rates for solutions to a semilinear wave equation with localized interior damping and a source term, subject to Neumann-type boundary condition.

A natural question arises in the context of the wave equation on compact surfaces: Would it be possible to stabilize the system by considering a localized feedback acting on a part of the surface? In affirmative case, what would be the geometrical impositions we have to assume on the surface? When the damping term acts on the whole surface, the conjecture was studied by Cavalcanti and Domingos Cavalcanti in [CA-DO] and also by Andrade et al. in [An1, An2] in the context of viscoelastic problems. However, as far as we are concerned, there is no result in the literature regarding the nonlinear wave equation on compact surfaces when the damping term acts in a portion $\mathcal{M}_{*}$ strictly contained in $\mathcal{M}$. For the linear case, we can mention the works due to Rauch and Taylor [Ra-Ta], Hitrik [HIT] and, more recently, Christianson [CHR].

The main goal of this paper is exactly to prove the above conjecture when the portion of $\mathcal{M}$, where the damping is effective is strategically chosen. For $i=1, \ldots, k$, assume that there exist open subsets $\mathcal{M}_{0 i} \subset \mathcal{M}_{0}$ of $\mathcal{M}$ with smooth boundary $\partial \mathcal{M}_{0 i}$ such that $\mathcal{M}_{0 i}$ are umbilical, or, more generally, that the principal curvatures $k_{1}$ and $k_{2}$ satisfy $\left|k_{1}(x)-k_{2}(x)\right|<\varepsilon_{i}\left(\varepsilon_{i}\right.$ considered small enough) for all $x \in \mathcal{M}_{0 i}$. Moreover, suppose that the mean curvature $H$ of each $\mathcal{M}_{0 i}$ is non-positive (i.e. $H \leq 0$ on $\mathcal{M}_{0 i}$ for every $i=1, \ldots, k)$ and that the damping is effective on an open subset $\mathcal{M}_{*} \subset \mathcal{M}$ that contains $\mathcal{M} \backslash \cup_{i=1}^{k} \mathcal{M}_{0 i}$.

The strategy used to prove the above conjecture is basically to make use of multipliers and fields as in Lions [LIONS1] with new ingredients that will be clarified in section 4. Indeed, the main difficulty and the novelty in these kind of problems on surfaces is how to deal with (or to interpret) the new terms which appear in the computations that come from the geometrical structure of $\mathcal{M}$. Moreover, this approach 
can be naturally extended for semilinear wave equation where the semilinear function $f(s)$ is assumed to be super-linear. We would like to emphasize that the proofs of [Ra-Ta, BAR, HIT], based on microlocal analysis, do not extend to the nonlinear problem (1.2). In addition, making use of arguments due to Lasiecka and Tataru [LA-TA] we obtain optimal decay rates of the energy. The obtained decay rates are optimal, since when we are able to explicit them (as in Cavalcanti, Domingos Cavalcanti and Lasiecka [CA-DO-LA]), they are the same as these optimal rates derived in the recent works of Alabau-Boussouira [ALA] or Toudykov [Tou].

Our paper is organized as follows. Section 2 is concerned with the statement of the problem and we introduce some notation. Our main result is stated in Section 3. Section 4 is devoted to the proof of the main result.

2. Statement of Problem. Let $\mathcal{M}$ be a smooth oriented embedded compact surface without boundary in $\mathbb{R}^{3}$ with $\mathcal{M}=\mathcal{M}_{0} \cup \mathcal{M}_{1}$, where

$$
\mathcal{M}_{1}:=\{x \in \mathcal{M} ; m(x) \cdot \nu(x)>0\} \quad, \text { AND } \mathcal{M}_{0}=\mathcal{M} \backslash \mathcal{M}_{1} .
$$

Here, $m$ is the vector field defined by $m(x):=x-x^{0},\left(x^{0} \in \mathbb{R}^{3}\right.$ fixed) and $\nu$ is the exterior unit normal vector field of $\mathcal{M}$.

In this paper, we investigate the stability properties of functions $\left[u(x, t), u_{t}(x, t)\right]$ which solve the following damped problem:

$$
\left\{\begin{array}{l}
\left.u_{t t}-\Delta_{\mathcal{M}} u+a(x) g\left(u_{t}\right)=0 \quad \text { on } \mathcal{M} \times\right] 0, \infty[, \\
u(0)=u^{0}, \quad u_{t}(0)=u^{1}
\end{array}\right.
$$

where the feedback function $g$ satisfies the following assumptions:

Assumption 2.1.

(i) $g(s)$ is continuous and monotone increasing,

(ii) $g(s) s>0$ for $s \neq 0$,

(iii) $k|s| \leq g(s) \leq K|s|$ for $|s|>1$,

where $k$ and $K$ are two positive constants.

In addition, to obtain the stabilization of problem (2.2), we shall need the following geometrical assumption:

Assumption 2.2. Remember that for $i=1, \ldots, k, \mathcal{M}_{0 i} \subset \mathcal{M}_{0}$ are open sets with smooth boundary $\partial \mathcal{M}_{0 i}$ such that $H \leq 0$ and $\mathcal{M}_{0 i}$ are umbilical submanifolds, or more generally, that the principal curvatures $k_{1}$ and $k_{2}$ satisfy $\left|k_{1}(x)-k_{2}(x)\right|<\varepsilon_{i}$ ( $\varepsilon_{i}$ considered small enough) for all $x \in \mathcal{M}_{0 i}$. We assume that $a \in L^{\infty}(\mathcal{M})$ is a nonnegative function such that

$$
a(x) \geq a_{0}>0, \quad \text { a. e. on } \mathcal{M}_{*},
$$

where $\mathcal{M}_{*}$ is an open set of $\mathcal{M}$ which contains $\mathcal{M} \backslash \cup_{i=1}^{k} \mathcal{M}_{0 i}$.

In order to fix ideas, Figure 1 shows a compact surface $\mathcal{M}$ such that there exists only one subset $\mathcal{M}_{01}$, which we take as the interior of $\mathcal{M}_{0}$.

In the sequel we define by $\Sigma=\mathcal{M} \times] 0, T\left[, \Sigma_{i}=\mathcal{M}_{i} \times\right] 0, T[, i=0,1$.

Let us considerer the Sobolev spaces $H^{s}(\mathcal{M}), s \in \mathbb{R}$ as in Lions and Magenes [LiMa] section 7.3. 


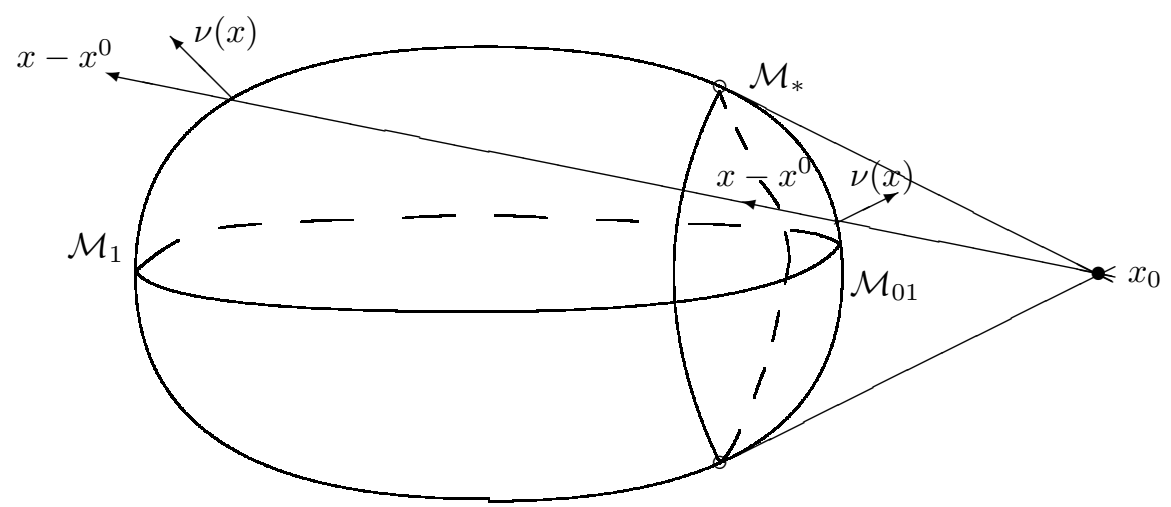

FIG. 1. The observer is at $x_{0}$. The subset $\mathcal{M}_{0}$ is the "visible" part of $\mathcal{M}$ and $\mathcal{M}_{1}$ is its complement. The subset $\mathcal{M}_{*} \supset \mathcal{M} \backslash \cup_{i=1}^{k} \mathcal{M}_{0 i}=\mathcal{M} \backslash \mathcal{M}_{01}$ is an open set that contains $\mathcal{M} \backslash \cup_{i=1}^{k} \mathcal{M}_{0 i}$ and the damping is effective there.

On the other hand, by using the Laplace-Beltrami operator $\Delta_{\mathcal{M}}$ on $\mathcal{M}$, we can give a more intrinsic definition of the spaces $H^{s}(\mathcal{M})$, by considering

$$
H^{2 m}(\mathcal{M})=\left\{u \in L^{2}(\mathcal{M}) / \Delta_{\mathcal{M}}^{m} u \in L^{2}(\mathcal{M})\right\}
$$

which, equipped with the canonical norm

$$
\|u\|_{H^{2 m}(\mathcal{M})}^{2}=\|u\|_{L^{2}(\mathcal{M})}^{2}+\left\|\Delta_{\mathcal{M}}^{m} u\right\|_{L^{2}(\mathcal{M})}^{2}
$$

is a Hilbert space.

We set

$$
V:=\left\{v \in H^{1}(\mathcal{M}) ; \int_{\mathcal{M}} v(x) d \mathcal{M}=0\right\}
$$

which is a Hilbert space endowed with the topology given by $H^{1}(\mathcal{M})$.

The condition $\int_{\mathcal{M}} v(x) d \mathcal{M}=0$ is required in order to guarantee the validity of the Poincaré inequality,

$$
\|f\|_{L^{2}(\mathcal{M})}^{2} \leq\left(\lambda_{1}\right)^{-1}\left\|\nabla_{T} f\right\|_{L^{2}(\mathcal{M})}^{2}, \quad \text { for all } f \in H^{1}(\mathcal{M}),
$$

where $\lambda_{1}$ is the first eigenvalue of the Laplace-Beltrami operator.

We observe that the problem (2.2) can be written in the following form

$$
\frac{d U}{d t}+\mathcal{A} U=G(U)
$$

where

$$
U=\left(\begin{array}{c}
u \\
u_{t}
\end{array}\right) \text { and } \mathcal{A}=\left(\begin{array}{cc}
0 & -I \\
-\Delta_{\mathcal{M}} & 0
\end{array}\right)
$$

is a maximal monotone operator and $G(\cdot)$ represents a locally Lipschitz perturbation. So, making use of standard semigroup arguments we have the following result: 
THEOREM 2.1 .

- (i) Under the conditions above, problem (2.2) is well posed in the space $V \times$ $L^{2}(\mathcal{M})$, i.e. for any initial data $\left\{u^{0}, u^{1}\right\} \in V \times L^{2}(\mathcal{M})$, there exists a unique weak solution of (2.2) in the class

$$
u \in C\left(\mathbb{R}_{+} ; V\right) \cap C^{1}\left(\mathbb{R}_{+} ; L^{2}(\mathcal{M})\right) .
$$

- (ii) In addition, the velocity term of the solution have the following regularity:

$$
u_{t} \in L_{l o c}^{2}\left(\mathbb{R}_{+} ; L^{2}(\mathcal{M})\right)
$$

(consequently, $g\left(u_{t}\right) \in L_{\text {loc }}^{2}\left(\mathbb{R}_{+} ; L^{2}(\mathcal{M})\right)$ by Assumption 2.1.

Furthermore, if $\left\{u^{0}, u^{1}\right\} \in\left\{V \cap H^{2}(\mathcal{M}) \times V\right\}$ then the solution has the following regularity

$$
u \in L^{\infty}\left(\mathbb{R}_{+} ; V \cap H^{2}(\mathcal{M})\right) \cap W^{1, \infty}\left(\mathbb{R}_{+} ; V\right) \cap W^{2, \infty}\left(\mathbb{R}_{+} ; L^{2}(\mathcal{M})\right) .
$$

REMARK 2.1. It is convenient to observe that the space $V$ may be not invariant under the flow because of the nonlinear character of the equation. In this case it is sufficient to add an extra term $\alpha u,(\alpha>0)$ in the equation in order to control $L_{2}$ norms. However, for simplicity in the computations, we shall omit this term since it does not bring any additional difficulty.

Supposing that $u$ is the unique global weak solution of problem (2.2), we define the corresponding energy functional by

$$
E(t)=\frac{1}{2} \int_{\mathcal{M}}\left[\left|u_{t}(x, t)\right|^{2}+\left|\nabla_{T} u(x, t)\right|^{2}\right] d \mathcal{M}
$$

For every solution of (2.2) in the class (2.6) the following identity holds

$$
E\left(t_{2}\right)-E\left(t_{1}\right)=-\int_{t_{1}}^{t_{2}} \int_{\mathcal{M}} a(x) g\left(u_{t}\right) u_{t} d \mathcal{M} d t, \text { for all } t_{2}>t_{1} \geq 0
$$

and therefore the energy is a non increasing function of the time variable $t$.

3. Main Result. Before stating our stability result, we will define some needed functions. For this purpose, we are following the ideas firstly introduced in Lasiecka and Tataru [LA-TA]. For the reader's comprehension we will repeat them briefly. Let $h$ be a concave, strictly increasing function, with $h(0)=0$, and such that

$$
h(s g(s))) \geq s^{2}+g^{2}(s), \text { for }|s| \leq 1 .
$$

Note that such function can be straightforwardly constructed, given the hypotheses on $g$ in Assumption 2.1. With this function, we define

$$
r(.)=h\left(\frac{\cdot}{\text { meas }\left(\Sigma_{1}\right)}\right) .
$$

As $r$ is monotone increasing, then $c I+r$ is invertible for all $c \geq 0$. For $L$ a positive constant, we set

$$
p(x)=(c I+r)^{-1}(L x),
$$


where the function $p$ is easily seen to be positive, continuous and strictly increasing with $p(0)=0$. Finally, let

$$
q(x)=x-(I+p)^{-1}(x) .
$$

We are now able to state our stability result.

Theorem 3.1. Assume that Assumption 2.1 and Assumption 2.2 are in place. Let $u$ be the weak solution of the problem (2.2). With the energy $E(t)$ defined as in (2.8), there exists a $T_{0}>0$ such that

$$
E(t) \leq S\left(\frac{t}{T_{0}}-1\right), \quad \forall t>T_{0},
$$

with $\lim _{t \rightarrow \infty} S(t)=0$, where the contraction semigroup $S(t)$ is the solution of the differential equation

$$
\frac{d}{d t} S(t)+q(S(t))=0, \quad S(0)=E(0),
$$

(where $q$ is given in (3.4)). Here, the constant $L$ (from definition (3.3)) will depend on meas $(\Sigma)$, and the constant $c$ (from definition (3.3)) is taken here to be $c \equiv \frac{k^{-1}+K}{\operatorname{meas}(\Sigma)\left(1+\|a\|_{\infty}\right)}$.

REMARK 3.1. If the feedback is linear, e. $g ., g(s)=s$, then, under the same assumptions as in Theorem 3.1, we have that the energy of problem (2.2) decays exponentially with respect to the initial energy. There exist two positive constants $C>0$ and $k>0$ such that

$$
E(t) \leq C e^{-k t} E(0), \quad t>0 .
$$

As another example, we can consider $g(s)=s^{p}, p>1$ at the origin. Since the function $s^{\frac{p+1}{2}}$ is convex for $p \geq 1$, then solving

$$
S_{t}+S^{\frac{p+1}{2}}=0
$$

we obtain the following polynomial decay rate:

$$
E(t) \leq C(E(0))\left[E(0)^{\frac{-p+1}{2}}+t(p-1)\right]^{\frac{2}{-p+1}} .
$$

We can find more interesting explicit decay rates in Cavalcanti, Domingos Cavalcanti and Lasiecka [CA-DO-LA].

\section{Proof of Main result.}

4.1. Preliminaries. We collect, below, some few formulas to be invoked in the sequel.

Let $\nu$ be the exterior normal vector field on $\mathcal{M}$. For all $x \in \mathcal{M}$, we denote by $\pi(x)$ the orthogonal projection on the tangent plane $T_{x} \mathcal{M}$. Any regular vector field $q: \mathbb{R}^{3} \rightarrow \mathbb{R}^{3}$ will be split up as follows:

$$
q(x)=q_{T}+(q(x) \cdot \nu(x)) \nu(x),
$$


where $q_{T}=\pi(x) q(x)$ is the tangential component of $q$.

If $\varphi: \mathbb{R}^{3} \rightarrow \mathbb{R}$ is a regular function, we have

$$
\begin{gathered}
\nabla \varphi=\partial_{\nu} \varphi \nu+\nabla_{T} \varphi \quad \text { on } \mathcal{M}, \\
|\nabla \varphi|^{2}=\left|\partial_{\nu} \varphi\right|^{2}+\left|\nabla_{T} \varphi\right|^{2} \quad \text { on } \mathcal{M},
\end{gathered}
$$

where $\partial_{\nu}$ represents the normal derivative towards the exterior of $\mathcal{M}$ and $\nabla_{T} \varphi$ is the tangential gradient of $\varphi$.

The Laplace- Beltrami operator $\Delta_{\mathcal{M}}$ of a function $\varphi: \mathcal{M} \rightarrow \mathbb{R}$ of class $C^{2}$ is defined by

$$
\Delta_{\mathcal{M}} \varphi:=\operatorname{div}_{T} \nabla_{T} \varphi
$$

where $\operatorname{div}_{T} \nabla_{T} \varphi$, is the divergent of the vector field $\nabla_{T} \varphi$.

Assuming that $\varphi: \mathcal{M} \rightarrow \mathbb{R}$ is a function of class $C^{1}$ and $q: \mathbb{R}^{3} \rightarrow \mathbb{R}^{3}$ is a vector field of class $C^{1}$, we have,

$$
\begin{gathered}
\int_{\mathcal{M}} q_{T} \cdot \nabla_{T} \varphi d \mathcal{M}=-\int_{\mathcal{M}} \operatorname{div}_{T} \varphi d \mathcal{M}, \\
2 \varphi\left(q_{T} \cdot \nabla_{T} \varphi\right)=q_{T} \cdot \nabla_{T}\left(\varphi^{2}\right) .
\end{gathered}
$$

From (4.5) and (4.6), we conclude the following formula

$$
2 \int_{\mathcal{M}} \varphi\left(q_{T} \cdot \nabla_{T} \varphi\right) d \mathcal{M}=\int_{\mathcal{M}} q_{T} \cdot \nabla_{T}\left(\varphi^{2}\right) d \mathcal{M}=-\int_{\mathcal{M}} \operatorname{div}_{T} q_{T}|\varphi|^{2} d \mathcal{M} .
$$

We observe that in the particular case when $m(x)=x-x^{0}, x \in \mathbb{R}^{3}$ and $x^{0} \in \mathbb{R}^{3}$ is a fixed point in $\mathbb{R}^{3}$, we have

$$
\operatorname{div} m=3, \quad \operatorname{div}_{T} m_{T}=2+(m \cdot \nu) \operatorname{Tr} B .
$$

where $B$ is the second fundamental form of $\mathcal{M}$ (the shape operator) and $\operatorname{Tr}$ is the trace. Let $\varphi$ and $m$ defined as above. We also have,

$$
\nabla_{T} \varphi \cdot \nabla_{T} m_{T} \cdot \nabla_{T} \varphi=\left|\nabla_{T} \varphi\right|^{2}+(m \cdot \nu)\left(\nabla_{T} \varphi \cdot B \cdot \nabla_{T} \varphi\right) .
$$

The proof of the above formulas can be found in Nedelec [NE], Lemrabet [LEM1], Heminna [HEM3] and references therein.

REMARK 4.1. The sign of $B$ can change in the literature. In our case, we remember that $B=-d N$, where $N$ is the Gauss map related to $\nu$.

The formula (4.8) can be rewritten by

$$
\operatorname{div} m=3, \quad \operatorname{div}_{T} m_{T}=2+2 H(m \cdot \nu) .
$$

where $H=\frac{\operatorname{tr} B}{2}$ is the mean curvature of $\mathcal{M}$.

We define a continuous linear operator $-\Delta_{\tilde{\mathcal{M}}}: H^{1}(\tilde{\mathcal{M}}) \rightarrow\left(H^{1}(\tilde{\mathcal{M}})\right)^{\prime}$, where $\tilde{\mathcal{M}}$ is a nomempty open subset of $\mathcal{M}$ (sometimes the whole $\mathcal{M}$ ) such that

$$
\left\langle-\Delta_{\tilde{\mathcal{M}}} \varphi, \psi\right\rangle=\int_{\tilde{\mathcal{M}}} \nabla_{T} \varphi \cdot \nabla_{T} \psi d \mathcal{M}, \quad \forall \varphi, \psi \in H^{1}(\tilde{\mathcal{M}})
$$


and, in particular,

$$
\left\langle-\Delta_{\tilde{\mathcal{M}}} \varphi, \varphi\right\rangle=\int_{\tilde{\mathcal{M}}}\left|\nabla_{T} \varphi\right|^{2} d \mathcal{M}, \quad \forall \varphi \in H^{1}(\tilde{\mathcal{M}}) .
$$

The operator $-\Delta_{\tilde{\mathcal{M}}}+I$ defines an isomorphism from $H^{1}(\tilde{\mathcal{M}})$ over $\left[H^{1}(\tilde{\mathcal{M}})\right]^{\prime}$. We observe that when $\tilde{\mathcal{M}}$ is a manifold without boundary, and this is the case, for instance, if $\tilde{\mathcal{M}}=\mathcal{M}$, we have $H^{1}(\tilde{\mathcal{M}})=H_{0}^{1}(\tilde{\mathcal{M}})$ and, consequently, $\left[H^{1}(\tilde{\mathcal{M}})\right]^{\prime}=H^{-1}(\tilde{\mathcal{M}})$.

REMARK 4.2. It is convenient to observe that all the classical formulas above stated can be extended for Sobolev spaces by using density arguments.

The proof of Theorem 3.1 proceeds through several steps.

4.2. An identity. We begin by proving the following proposition

Proposition 4.2.1. Let $\mathcal{M} \subset \mathbb{R}^{3}$ be an oriented regular compact surface without boundary and $q$ a vector field with $q=q_{T}+(q \cdot \nu) \nu$. Then, for every regular solution u of (1.2) we have the following identity

$$
\begin{aligned}
& {\left[\int_{\mathcal{M}} u_{t} q_{T} \cdot \nabla_{T} u d \mathcal{M}\right]_{0}^{T}+\frac{1}{2} \int_{0}^{T} \int_{\mathcal{M}}\left(\operatorname{div}_{T} q_{T}\right)\left\{\left|u_{t}\right|^{2}-\left|\nabla_{T} u\right|^{2}\right\} d \mathcal{M} d t} \\
& +\int_{0}^{T} \int_{\mathcal{M}} \nabla_{T} u \cdot \nabla_{T} q_{T} \cdot \nabla_{T} u d \mathcal{M} d t+\int_{0}^{T} \int_{\mathcal{M}} a(x) g\left(u_{t}\right)\left(q_{T} \cdot \nabla_{T} u\right) d \mathcal{M} d t=0 .
\end{aligned}
$$

Proof. Multiplying the equation of (1.2) by the multiplier $q_{T} \cdot \nabla_{T} u$ and integrating on $\mathcal{M} \times] 0, T[$, we obtain

$$
0=\int_{0}^{T} \int_{\mathcal{M}}\left(u_{t t}-\Delta_{\mathcal{M}} u+a(x) g\left(u_{t}\right)\right)\left(q_{T} \cdot \nabla_{T} u\right) d \mathcal{M} d t .
$$

Next, we will estimate some terms on the RHS of identity (4.14). Taking (4.11), (4.6) and (4.7) into account, we obtain

$$
\begin{aligned}
& \int_{0}^{T} \int_{\mathcal{M}}\left(-\Delta_{\mathcal{M}} u\right)\left(q_{T} \cdot \nabla_{T} u\right) d \mathcal{M} d t=\int_{0}^{T} \int_{\mathcal{M}} \nabla_{T} u \cdot \nabla_{T}\left(q_{T} \cdot \nabla_{T} u\right) d \mathcal{M} d t \\
& =\int_{0}^{T} \int_{\mathcal{M}} \nabla_{T} u \cdot \nabla_{T} q_{T} \cdot \nabla_{T} u d \mathcal{M} d t+\frac{1}{2} \int_{0}^{T} \int_{\mathcal{M}} q_{T} \cdot \nabla_{T}\left[\left|\nabla_{T} u\right|^{2}\right] d \mathcal{M} d t \\
& =\int_{0}^{T} \int_{\mathcal{M}} \nabla_{T} u \cdot \nabla_{T} q_{T} \cdot \nabla_{T} u d \mathcal{M} d t-\frac{1}{2} \int_{0}^{T} \int_{\mathcal{M}}\left|\nabla_{T} u\right|^{2} d i v_{T} q_{T} d \mathcal{M} d t
\end{aligned}
$$

and, integrating by parts and considering (4.7), we obtain

$$
\begin{aligned}
& \int_{0}^{T} \int_{\mathcal{M}}\left(u_{t t}+a(x) g\left(u_{t}\right)\right)\left(q_{T} \cdot \nabla_{T} u\right) d \mathcal{M} d t \\
= & {\left[\int_{\mathcal{M}} u_{t}\left(q_{T} \cdot \nabla_{T} u\right)\right]_{0}^{T}-\int_{0}^{T} \int_{\mathcal{M}} u_{t}\left(q_{T} \cdot \nabla_{T} u_{t}\right) d \mathcal{M} d t } \\
& +\int_{0}^{T} \int_{\mathcal{M}} a(x) g\left(u_{t}\right)\left(q_{T} \cdot \nabla_{T} u\right) d \mathcal{M} d t \\
= & {\left[\int_{\mathcal{M}} u_{t}\left(q_{T} \cdot \nabla_{T} u\right)\right]_{0}^{T}+\frac{1}{2} \int_{0}^{T} \int_{\mathcal{M}}\left(d_{i} v_{T} q_{T}\right)\left|u_{t}\right|^{2} d \mathcal{M} d t } \\
& +\int_{0}^{T} \int_{\mathcal{M}} a(x) g\left(u_{t}\right)\left(q_{T} \cdot \nabla_{T} u\right) d \mathcal{M} d t .
\end{aligned}
$$


Combining (4.14), (4.15) and (4.16), we deduce (4.13), which concludes the proof of Proposition 4.2.1. $\square$

Employing (4.13) with $q(x)=m(x)=x-x^{0}$ for some $x^{0} \in \mathbb{R}^{3}$ fixed and taking (4.8) and (4.9) into account, we infer

$$
\begin{aligned}
& {\left[\int_{\mathcal{M}} u_{t} m_{T} \cdot \nabla_{T} u d \mathcal{M}\right]_{0}^{T}+\int_{0}^{T} \int_{\mathcal{M}}\left\{\left|u_{t}\right|^{2}-\left|\nabla_{T} u\right|^{2}\right\} d \mathcal{M} d t} \\
& +\int_{0}^{T} \int_{\mathcal{M}}\left[\left|\nabla_{T} u\right|^{2}+(m \cdot \nu)\left(\nabla_{T} u \cdot B \cdot \nabla_{T} u\right)\right] d \mathcal{M} d t \\
& +\int_{0}^{T} \int_{\mathcal{M}}(m \cdot \nu) H\left\{\left|u_{t}\right|^{2}-\left|\nabla_{T} u\right|^{2}\right\} d \mathcal{M} d t \\
& +\int_{0}^{T} \int_{\mathcal{M}} a(x) g\left(u_{t}\right)\left(m_{T} \cdot \nabla_{T} u\right) d \mathcal{M} d t=0 .
\end{aligned}
$$

We have the following identity:

Lemma 4.2.3. Let $u$ be a weak solution to problem (1.2) and $\xi \in C^{1}(\mathcal{M})$. Then

$$
\begin{aligned}
{\left[\int_{\mathcal{M}} u_{t} \xi u d \mathcal{M}\right]_{0}^{T} } & =\int_{0}^{T} \int_{\mathcal{M}} \xi\left|u_{t}\right|^{2} d \mathcal{M} d t-\int_{0}^{T} \int_{\mathcal{M}} \xi\left|\nabla_{T} u\right|^{2} d \mathcal{M} d t \\
& -\int_{0}^{T} \int_{\mathcal{M}}\left(\nabla_{T} u \cdot \nabla_{T} \xi\right) u d \mathcal{M} d t-\int_{0}^{T} \int_{\mathcal{M}} a(x) g\left(u_{t}\right) \xi u d \mathcal{M} d t
\end{aligned}
$$

Proof. Multiplying the first equation of (1.2) by $\xi u$ and integrating by parts we obtain the desired. $\square$

Substituting $\xi=\frac{1}{2}$ in (4.18) and combining the obtained result with identity (4.17) we deduce

$$
\begin{aligned}
& {\left[\int_{\mathcal{M}} u_{t} m_{T} \cdot \nabla_{T} u d \mathcal{M}\right]_{0}^{T}+\frac{1}{2}\left[\int_{\mathcal{M}} u_{t} u d \mathcal{M}\right]_{0}^{T}} \\
& +\int_{0}^{T} E(t) d t+\int_{0}^{T} \int_{\mathcal{M}} a(x) g\left(u_{t}\right)\left(m_{T} \cdot \nabla_{T} u\right) d \mathcal{M} d t \\
& +\frac{1}{2} \int_{0}^{T} \int_{\mathcal{M}} a(x) g\left(u_{t}\right) u d \mathcal{M} d t \\
& =-\int_{0}^{T} \int_{\mathcal{M}}(m \cdot \nu) H\left\{\left|u_{t}\right|^{2}-\left|\nabla_{T} u\right|^{2}\right\} d \mathcal{M} d t . \\
& -\int_{0}^{T} \int_{\mathcal{M}}(m \cdot \nu)\left(\nabla_{T} u \cdot B \cdot \nabla_{T} u\right) d \mathcal{M} d t .
\end{aligned}
$$

\section{Analysis of the terms which involve the shape operator $B$}

Let us focus our attention on the shape operator $B: T_{x} \mathcal{M} \rightarrow T_{x} \mathcal{M}$. There exist an orthonormal basis $\left\{e_{1}, e_{2}\right\}$ of $T_{x} \mathcal{M}$ such that $B e_{1}=k_{1} e_{1}$ and $B e_{2}=k_{2} e_{2} . k_{1}$ and $k_{2}$ are the principal curvatures of $\mathcal{M}$ at $x$. The matrix of $B$ with respect to the basis $\left\{e_{1}, e_{2}\right\}$ is given by 


$$
B:=\left(\begin{array}{cc}
k_{1} & 0 \\
0 & k_{2}
\end{array}\right) .
$$

Setting $\nabla_{T} u=(\xi, \eta)$ the coordinates of $\nabla_{T} u$ in the basis $\left\{e_{1}, e_{2}\right\}$, for each $x \in \mathcal{M}$, we deduce that

$$
\nabla_{T} u \cdot B \cdot \nabla_{T} u=k_{1} \xi^{2}+k_{2} \eta^{2}
$$

Then, from (4.20), we infer

$$
\begin{aligned}
& (m \cdot \nu)\left[\left(\nabla_{T} u \cdot B \cdot \nabla_{T} u\right)-\frac{1}{2} \operatorname{Tr}(B)\left|\nabla_{T} u\right|^{2}\right] \\
& =(m \cdot \nu)\left[\frac{\left(k_{1}-k_{2}\right)}{2} \xi^{2}+\frac{\left(k_{2}-k_{1}\right)}{2} \eta^{2}\right] .
\end{aligned}
$$

REMARK 4.3. Observe that this is the precise moment that the intrinsic properties of the manifold $\mathcal{M}$ appear, that is, we strongly need that the term $-\int_{0}^{T} \int_{\mathcal{M}}(m$. $\nu) H u_{t}^{2} d \mathcal{M} d t$ lies in a region where the damping term is effective. Remember that the damping term is effective on an open set $\mathcal{M}_{*}$ which contains $\mathcal{M} \backslash \cup_{i=1}^{k} \mathcal{M}_{0 i}$. So, assuming that $H \leq 0$ and since $m(x) \cdot \nu(x) \leq 0$ on $\mathcal{M}_{0}$, we have

$$
-\int_{0}^{T} \int_{\mathcal{M}_{0}}(m \cdot \nu) H\left|u_{t}\right|^{2} d \mathcal{M} d t \leq 0
$$

In addition, supposing that $\mathcal{M}_{0 i}$ is umbilical for every $i=1, \ldots, k$, then, having (4.21) in mind, we also have that

$$
\int_{0}^{T} \int_{\mathcal{M}_{0 i}}(m \cdot \nu)\left[H\left|\nabla_{T} u\right|^{2}-\left(\nabla_{T} u \cdot B \cdot \nabla_{T} u\right)\right] d \mathcal{M} d t=0, \quad i=1, \ldots, k .
$$

More generally, assuming that the principal curvatures $k_{1}$ and $k_{2}$ satisfy $\mid k_{1}(x)-$ $k_{2}(x) \mid<\varepsilon_{i}$ (here, $\varepsilon_{i}$ is assumed sufficiently small) for all $x \in \mathcal{M}_{0 i}, i=1, \cdots, k$, we deduce that

$$
\begin{aligned}
& \left|\sum_{i=1}^{k} \int_{0}^{T} \int_{\mathcal{M}_{0 i}}(m \cdot \nu)\left[H\left|\nabla_{T} u\right|^{2}-\left(\nabla_{T} u \cdot B \cdot \nabla_{T} u\right)\right] d \mathcal{M} d t\right| \\
& \leq \sum_{i=1}^{k} \int_{0}^{T} \int_{\mathcal{M}_{0 i}}|(m \cdot \nu)|\left|k_{1}-k_{2}\right|\left|\xi^{2}+\eta^{2}\right| d \mathcal{M} d t \\
& \leq \sum_{i=1}^{k} R_{i} \varepsilon_{i} \int_{0}^{T} \int_{\mathcal{M}_{0 i}}\left|\nabla_{T} u\right|^{2} d \mathcal{M} d t \leq 2 \sum_{i=1}^{k} R_{i} \varepsilon_{i} \int_{0}^{T} E(t) d t,
\end{aligned}
$$

where $R_{i}=\max _{x \in \overline{\mathcal{M}_{0 i}}}\left\|x-x^{0}\right\|_{\mathbb{R}^{3}}$.

Set $\mathcal{M}_{2}=\mathcal{M} \backslash \cup_{i=1}^{k} \mathcal{M}_{0 i}$. In the case where $\mathcal{M}_{0 i}$ are umbilical, recalling (4.19) 
taking (4.21) and Remark 4.3 into consideration, we deduce

$$
\begin{aligned}
\int_{0}^{T} E(t) d t & \leq-\left[\int_{\mathcal{M}} u_{t} m_{T} \cdot \nabla_{T} u d \mathcal{M}\right]_{0}^{T}-\frac{1}{2}\left[\int_{\mathcal{M}} u_{t} u d \mathcal{M}\right]_{0}^{T} \\
& +\int_{0}^{T} \int_{\mathcal{M}_{2}}(m \cdot \nu)\left[H\left|\nabla_{T} u\right|^{2}-\left(\nabla_{T} u \cdot B \cdot \nabla_{T} u\right)\right] d \mathcal{M} d t \\
& -\int_{0}^{T} \int_{\mathcal{M}_{2}}(m \cdot \nu) H\left|u_{t}\right|^{2} d \mathcal{M} d t \\
& -\int_{0}^{T} \int_{\mathcal{M}} a(x) g\left(u_{t}\right)\left(m_{T} \cdot \nabla_{T} u\right) d \mathcal{M} d t \\
& -\frac{1}{2} \int_{0}^{T} \int_{\mathcal{M}} a(x) g\left(u_{t}\right) u d \mathcal{M} d t .
\end{aligned}
$$

In the general case, the unique difference in the proof is that the term $\int_{0}^{T} E(t) d t$ that appears on the LHS of (4.22) will be multiplied by a positive constant $C$, provided that we consider $\varepsilon_{i}$ small enough. For simplicity we shall assume that $C=1$.

We will denote

$$
\chi=\left[\int_{\mathcal{M}} u_{t} m_{T} \cdot \nabla_{T} u d \mathcal{M}\right]_{0}^{T}+\frac{1}{2}\left[\int_{\mathcal{M}} u_{t} u d \mathcal{M}\right]_{0}^{T} .
$$

Next we will estimate some terms in (4.22). Let us denote:

$$
R:=\max _{x \in \mathcal{M}}\|m(x)\|_{\mathbb{R}^{n}}=\max _{x \in \mathcal{M}}\left\|x-x^{0}\right\|_{\mathbb{R}^{n}} .
$$

Estimate for $I_{1}:=\int_{0}^{T} \int_{\mathcal{M}} a(x) g\left(u_{t}\right)\left(m_{T} \cdot \nabla_{T} u\right) d \mathcal{M} d t$.

By Cauchy-Schwarz inequality, taking (4.24) into account and considering the inequality $a b \leq \frac{a^{2}}{4 \eta}+\eta b^{2}$, where $\eta$ is a positive number, we obtain

$$
\left|I_{1}\right| \leq \frac{\|a\|_{L^{\infty}(\mathcal{M})} R^{2}}{\eta} \int_{0}^{T} \int_{\mathcal{M}} a(x)\left|g\left(u_{t}\right)\right|^{2} d \mathcal{M} d t+2 \eta \int_{0}^{T} E(t) d t .
$$

Estimate for $I_{2}=\frac{1}{2} \int_{0}^{T} \int_{\mathcal{M}} a(x) g\left(u_{t}\right) u d \mathcal{M} d t$.

Similarly we infer

$$
\left|I_{2}\right| \leq \frac{\|a\|_{L^{\infty}(\mathcal{M})} \lambda_{1}^{-1}}{16 \eta} \int_{0}^{T} \int_{\mathcal{M}} a(x)\left|g\left(u_{t}\right)\right|^{2} d \mathcal{M} d t+2 \eta \int_{0}^{T} E(t) d t,
$$

where $\lambda_{1}$ comes from the Poincaré inequality given in (2.5).

Choosing $\eta=1 / 8$ and inserting (4.23), (4.25) and (4.26) into (4.22) yields

$$
\begin{aligned}
\frac{1}{2} \int_{0}^{T} E(t) d t & \leq|\chi|+C_{1} \int_{0}^{T} \int_{\mathcal{M}} a(x)\left(g\left(u_{t}\right)\right)^{2} d \mathcal{M} d t \\
& +C_{1} \int_{0}^{T} \int_{\mathcal{M}_{2}}\left[\left|\nabla_{T} u\right|^{2}+a(x) u_{t}^{2}\right] d \mathcal{M} d t
\end{aligned}
$$

where

$$
C_{1}:=\max \left\{\|a\|_{L^{\infty}(\mathcal{M})}\left[2^{-1} \lambda_{1}^{-1}+8 R^{2}\right], \| B|| R+|H| R, R|H| a_{0}^{-1}\right\},
$$


$\| B||=\sup _{x \in \mathcal{M}}\left|B_{x}\right|$, and $\left|B_{x}\right|=\sup _{\left\{v \in T_{x} \mathcal{M} ;|v|=1\right\}}\left|B_{x} v\right|$.

It remains to estimate the quantity $\int_{0}^{T} \int_{\mathcal{M}_{2}}\left|\nabla_{T} u\right|^{2} d \mathcal{M} d t$ in terms of the damping term $\int_{0}^{T} \int_{\mathcal{M}}\left[a(x)\left|g\left(u_{t}\right)\right|^{2}+a(x)\left|u_{t}\right|^{2}\right] d \mathcal{M} d t$. For this purpose we have to built a "cutoff" function $\eta_{\varepsilon}$ on a specific neighborhood of $\mathcal{M}_{2}$. First of all, define $\tilde{\eta}: \mathbb{R} \rightarrow \mathbb{R}$ such that

$$
\tilde{\eta}(x)=\left\{\begin{array}{ccc}
1 & \text { if } & x \leq 0 \\
(x-1)^{2} & \text { if } & x \in[1 / 2,1] \\
0 & \text { if } & x>1
\end{array}\right.
$$

and it is defined on $(0,1 / 2)$ in such a way that $\tilde{\eta}$ is a non-decreasing function of class $C^{1}$. For $\varepsilon>0$, set $\tilde{\eta}_{\varepsilon}(x):=\tilde{\eta}(x / \varepsilon)$. It is straightforward that there exist a constant $M$ which does not depend on $\varepsilon$ such that

$$
\frac{\left|\tilde{\eta}_{\varepsilon}^{\prime}(x)\right|^{2}}{\tilde{\eta}_{\varepsilon}(x)} \leq \frac{M}{\varepsilon^{2}}
$$

for every $x<\varepsilon$.

Now, let $\varepsilon>0$ be such that

$$
\tilde{\omega}_{\varepsilon}:=\left\{x \in \mathcal{M} ; d\left(x, \bigcup_{i=1}^{k} \partial \mathcal{M}_{0 i}\right)<\varepsilon\right\}
$$

is a tubular neighborhood of $\bigcup_{i=1}^{k} \partial \mathcal{M}_{0 i}$ and $\omega_{\varepsilon}:=\tilde{\omega}_{\varepsilon} \cup \mathcal{M}_{2}$ is contained in $\mathcal{M}_{*}$. Define $\eta_{\varepsilon}: \mathcal{M} \rightarrow \mathbb{R}$ as

$$
\eta_{\varepsilon}(x)=\left\{\begin{array}{clc}
1 & \text { if } & x \in \mathcal{M}_{2} \\
\tilde{\eta}_{\varepsilon}\left(d\left(x, \mathcal{M}_{2}\right)\right) & \text { if } & x \in \omega_{\varepsilon} \backslash \mathcal{M}_{2} \\
0 & \text { otherwise. } &
\end{array}\right.
$$

It is straightforward that $\eta_{\varepsilon}$ is a function of class $C^{1}$ on $\mathcal{M}$ due to the smoothness of $\partial \mathcal{M}_{2}$ and $\partial \omega_{\varepsilon}$. Notice also that

$$
\frac{\left|\nabla_{T} \eta_{\varepsilon}(x)\right|^{2}}{\eta_{\varepsilon}(x)}=\frac{\left|\tilde{\eta}_{\varepsilon}^{\prime}\left(d\left(x, \mathcal{M}_{2}\right)\right)\right|^{2}}{\tilde{\eta}_{\varepsilon}\left(d\left(x, \mathcal{M}_{2}\right)\right)} \leq \frac{M}{\varepsilon^{2}}
$$

for every $x \in \omega_{\varepsilon} \backslash \mathcal{M}_{2}$. In particular, $\frac{\left|\nabla_{T} \eta_{\varepsilon}\right|^{2}}{\eta_{\varepsilon}} \in L^{\infty}\left(\omega_{\varepsilon}\right)$.

Taking $\xi=\eta_{\varepsilon}$ in the identity (4.18) we obtain

$$
\begin{aligned}
& \int_{0}^{T} \int_{\omega_{\varepsilon}} \eta_{\varepsilon}\left|\nabla_{T} u\right|^{2} d \mathcal{M} d t \\
= & -\left[\int_{\omega_{\varepsilon}} u_{t} u \eta_{\varepsilon} d \mathcal{M}\right]_{0}^{T}+\int_{0}^{T} \int_{\omega_{\varepsilon}} \eta_{\varepsilon}\left|u_{t}\right|^{2} d \mathcal{M} \\
- & \int_{0}^{T} \int_{\omega_{\varepsilon}} u\left(\nabla_{T} u \cdot \nabla_{T} \eta_{\varepsilon}\right) d \mathcal{M} d t-\int_{0}^{T} \int_{\omega_{\varepsilon}} a(x) g\left(u_{t}\right) u \eta_{\varepsilon} d \mathcal{M} d t .
\end{aligned}
$$

Next we will estimate the terms on the RHS of (4.29).

Estimate for $K_{1}:=\int_{0}^{T} \int_{\omega_{\varepsilon}} \eta_{\varepsilon}\left|u_{t}\right|^{2} d \mathcal{M} d t$ 
From (2.3), since $\eta_{\varepsilon} \leq 1$ and $\omega_{\varepsilon} \subset \mathcal{M}_{*}$, where the damping lies, we deduce

$$
K_{1} \leq a_{0}^{-1} \int_{0}^{T} \int_{\mathcal{M}} a(x) u_{t}^{2} d \mathcal{M} d t
$$

Estimate for $K_{2}:=-\int_{0}^{T} \int_{\omega_{\varepsilon}} a(x) g\left(u_{t}\right) u \eta_{\varepsilon} d \mathcal{M} d t$.

The Cauchy-Schwarz inequality, the inequality $a b \leq \frac{1}{4 \alpha} a^{2}+\alpha b^{2}$ and (2.5) yield

$$
\left|K_{2}\right| \leq \frac{\lambda_{1}^{-1}|| a \|_{L^{\infty}(\mathcal{M})}}{4 \alpha} \int_{0}^{T} \int_{\mathcal{M}} a(x)\left|g\left(u_{t}\right)\right|^{2} d \mathcal{M}+2 \alpha \int_{0}^{T} E(t) d t
$$

where $\alpha$ is an arbitrary positive constant.

Estimate for $K_{3}:=\int_{0}^{T} \int_{\omega_{\varepsilon}} u\left(\nabla_{T} u \cdot \nabla_{T} \eta_{\varepsilon}\right) d \mathcal{M} d t$.

Considering (4.28) and applying Cauchy-Schwarz inequality, we can write

$$
\begin{aligned}
\left|K_{3}\right| & \leq \frac{1}{2} \int_{0}^{T}\left[\int_{\omega_{\varepsilon}} \eta_{\varepsilon}\left|\nabla_{T} u\right|^{2} d \mathcal{M}+\int_{\omega_{\varepsilon}} \frac{\left|\nabla_{T} \eta_{\varepsilon}\right|^{2}}{\eta_{\varepsilon}}|u|^{2} d \mathcal{M}\right] d t \\
& \leq \frac{1}{2} \int_{0}^{T}\left[\int_{\omega_{\varepsilon}} \eta_{\varepsilon}\left|\nabla_{T} u\right|^{2} d \mathcal{M}+\frac{M}{\varepsilon^{2}} \int_{\omega_{\varepsilon}}|u|^{2} d \mathcal{M}\right] d t
\end{aligned}
$$

Combining (4.29)-(4.32) we arrive to the following inequality

$$
\begin{aligned}
\frac{1}{2} \int_{0}^{T} \int_{\omega_{\varepsilon}} \eta_{\varepsilon}\left|\nabla_{T} u\right|^{2} d \mathcal{M} d t & \leq|\mathcal{Y}|+\frac{\lambda_{1}^{-1}|| a \|_{L^{\infty}(\mathcal{M})}}{4 \alpha} \int_{0}^{T} \int_{\mathcal{M}} a(x)\left|g\left(u_{t}\right)\right|^{2} d \mathcal{M} \\
& +2 \alpha \int_{0}^{T} E(t) d t+\frac{M}{2 \varepsilon^{2}} \int_{0}^{T} \int_{\omega_{\varepsilon}}|u|^{2} d \mathcal{M} d t \\
& +a_{0}^{-1} \int_{0}^{T} \int_{\mathcal{M}} a(x) u_{t}^{2} d \mathcal{M} d t .
\end{aligned}
$$

where

$$
\mathcal{Y}:=-\left[\int_{\omega_{\varepsilon}} u_{t} u \eta_{\varepsilon} d \mathcal{M}\right]_{0}^{T}
$$

Thus, combining (4.33) and (4.27), having in mind that

$$
\frac{1}{2} \int_{0}^{T} \int_{\mathcal{M}_{2}}\left|\nabla_{T} u\right|^{2} d \mathcal{M} d t \leq \frac{1}{2} \int_{0}^{T} \int_{\omega_{\varepsilon}} \eta_{\varepsilon}\left|\nabla_{T} u\right|^{2} d \mathcal{M} d t
$$

and choosing $\alpha=1 / 16 C_{1}$ we deduce

$$
\begin{aligned}
& \frac{1}{4} \int_{0}^{T} E(t) d t \leq|\chi|+2 C_{1}|\mathcal{Y}| \\
& +\max \left\{C_{1}, 8 C_{1}^{2} \lambda_{1}^{-1}|| a \|_{L^{\infty}(\mathcal{M})}, 2 C_{1} a_{0}^{-1}\right\} \int_{0}^{T} \int_{\mathcal{M}}\left[a(x)\left|g\left(u_{t}\right)\right|^{2}+a(x)\left|u_{t}\right|^{2}\right] d \mathcal{M} d t \\
& +\frac{M C_{1}}{\varepsilon^{2}} \int_{0}^{T} \int_{\omega_{\varepsilon}}|u|^{2} d \mathcal{M} d t
\end{aligned}
$$


On the other hand, from (4.23), (4.34) and (2.9) the following estimate holds

$$
\begin{aligned}
|\chi|+2 C_{2}|\mathcal{Y}| & \leq C(E(0)+E(T)) \\
& =C\left[2 E(T)+\int_{0}^{T} \int_{\mathcal{M}} a(x) g\left(u_{t}\right) u_{t} d \mathcal{M}\right],
\end{aligned}
$$

where $C$ is a positive constant which depends also on $R$.

Then, (4.35) and (4.36) yield

$$
\begin{aligned}
T E(T) & \leq \int_{0}^{T} E(t) d t \\
& \leq C E(T)+C\left[\int_{0}^{T} \int_{\mathcal{M}}\left[a(x)\left|g\left(u_{t}\right)\right|^{2}+a(x)\left|u_{t}\right|^{2}\right] d \mathcal{M} d t\right] \\
& +C \int_{0}^{T} \int_{\omega_{\varepsilon}}|u|^{2} d \mathcal{M} d t,
\end{aligned}
$$

where $C$ is a positive constant which depends on $a_{0},\|a\|_{\infty}, \lambda_{1}, R,|H|,\|B\|$ and $\frac{M}{\varepsilon^{2}}$.

Our aim is to estimate the last term on the RHS of (4.37). In order to do this let us consider the following lemma, where $T_{0}$ is a positive constant which is sufficiently large for our purpose.

Lemma 4.1. Under the hypothesis of Theorem 3.1 and for all $T>T_{0}$, there exists a positive constant $C\left(T_{0}, E(0)\right)$ such that if $\left(u, u_{t}\right)$ is the solution of (1.2) with weak initial data, we have

$$
\int_{0}^{T} \int_{\mathcal{M}}|u|^{2} d \mathcal{M} d t \leq C\left(T_{0}, E(0)\right)\left\{\int_{0}^{T} \int_{\mathcal{M}}\left(a(x) g^{2}\left(u_{t}\right)+a(x) u_{t}^{2}\right) d \mathcal{M} d t\right\} 4
$$

Proof. We argue by contradiction. For simplicity we shall denote $u^{\prime}:=u_{t}$. Let us suppose that (4.38) is not verified and let $\left\{u_{k}(0), u_{k}^{\prime}(0)\right\}$ be a sequence of initial data where the corresponding solutions $\left\{u_{k}\right\}_{k \in \mathbb{N}}$ of $(1.2)$ with $E_{k}(0)$, which is assumed to be uniformly bounded in $k$, verifies

$$
\lim _{k \rightarrow+\infty} \frac{\int_{0}^{T}\left\|u_{k}(t)\right\|_{L^{2}(\mathcal{M})}^{2} d t}{\int_{0}^{T} \int_{\mathcal{M}}\left(a(x) g^{2}\left(u_{k}^{\prime}\right)+a(x) u_{k}^{\prime 2}\right) d \mathcal{M} d t}=+\infty
$$

that is

$$
\lim _{k \rightarrow+\infty} \frac{\int_{0}^{T} \int_{\mathcal{M}}\left(a(x) g^{2}\left(u_{k}^{\prime}\right)+a(x) u_{k}^{\prime 2}\right) d \mathcal{M} d t}{\int_{0}^{T}\left\|u_{k}(t)\right\|_{L^{2}(\mathcal{M})}^{2} d t}=0 .
$$

Since $E_{k}(t) \leq E_{k}(0) \leq L$, where $L$ is a positive constant, we obtain a subsequence, still denoted by $\left\{u_{k}\right\}$ from now on, which verifies the convergence:

$$
\begin{aligned}
& u_{k} \rightarrow u \text { weakly in } H^{1}\left(\Sigma_{T}\right), \\
& u_{k} \rightarrow u \text { weak star in } L^{\infty}(0, T ; V), \\
& u_{k}^{\prime} \rightarrow u^{\prime} \text { weak star in } L^{\infty}\left(0, T ; L^{2}(\mathcal{M})\right) .
\end{aligned}
$$


Employing compactness results we also deduce that

$$
u_{k} \rightarrow u \text { strongly in } L^{2}\left(0, T ; L^{2}(\mathcal{M})\right) .
$$

At this point we will divide our proof into two cases, namely: when $u \neq 0$ and $u=0$.

(i) Case (I): $u \neq 0$.

We also observe that from (4.40) and (4.44) we have

$$
\lim _{k \rightarrow+\infty} \int_{0}^{T} \int_{\mathcal{M}}\left(a(x) g^{2}\left(u_{k}^{\prime}\right)+a(x) u_{k}^{\prime 2}\right) d \mathcal{M} d t=0
$$

Passing to the limit in the equation, when $k \rightarrow+\infty$, we get,

$$
\left\{\begin{aligned}
u_{t t}-\Delta_{\mathcal{M}} u & =0 \text { on } \mathcal{M} \times(0, T) \\
u_{t} & =0 \text { on } \mathcal{M}_{*} \times(0, T),
\end{aligned}\right.
$$

and for $u_{t}=v$, we obtain, in the distributional sense

$$
\left\{\begin{aligned}
v_{t t}-\Delta_{\mathcal{M}} v= & 0 \text { on } \mathcal{M} \times(0, T), \\
v= & 0 \text { on } \mathcal{M}_{*} \times(0, T) .
\end{aligned}\right.
$$

From uniqueness results from the work of Triggiani and Yao [TRI-YAO], we conclude that $v \equiv 0$, that is, $u_{t}=0$ Returning to (4.46) we obtain the following elliptic equation for a.e. $t \in(0, T)$ given by

$$
\Delta_{\mathcal{M}} u=0 \text { on } \mathcal{M}
$$

which implies that $u=0$, which is a contradiction.

(ii) Case (II): $u=0$.

Defining

$$
c_{k}:=\left[\int_{0}^{T} \int_{\mathcal{M}}\left|u_{k}\right|^{2} d \mathcal{M} d t\right]^{1 / 2},
$$

and

$$
\bar{u}_{k}:=\frac{1}{c_{k}} u_{k}
$$

we obtain

$$
\int_{0}^{T} \int_{\mathcal{M}}\left|\bar{u}_{k}\right|^{2} d \mathcal{M} d t=\int_{0}^{T} \int_{\mathcal{M}} \frac{\left|u_{k}\right|^{2}}{c_{k}^{2}} d \mathcal{M} d t=\frac{1}{c_{k}^{2}} \int_{0}^{T} \int_{\mathcal{M}}\left|u_{k}\right|^{2} d \mathcal{M} d t=1 .
$$

Setting

$$
\bar{E}_{k}(t):=\frac{1}{2} \int_{\mathcal{M}}\left|\bar{u}_{k}^{\prime}\right|^{2} d \mathcal{M}+\frac{1}{2} \int_{\mathcal{M}}\left|\nabla \bar{u}_{k}\right|^{2} d \mathcal{M}
$$


we deduce automatically that

$$
\bar{E}_{k}(t)=\frac{E_{k}(t)}{c_{k}^{2}}
$$

Recalling (4.37) we obtain, for $T$ large enough, that

$$
E(T) \leq \hat{C}\left[\int_{0}^{T} \int_{\mathcal{M}}\left(a(x) g^{2}\left(u_{t}\right)+a(x) u_{t}^{2}\right) d \mathcal{M} d t+\int_{0}^{T} \int_{\mathcal{M}}|u|^{2} d \mathcal{M} d t\right] .
$$

Employing the identity $E(T)-E(0)=-\int_{0}^{T} \int_{\mathcal{M}} a(x) g\left(u_{t}\right) u_{t} d \mathcal{M} d t$, we can write

$$
E(t) \leq E(0) \leq \tilde{C}\left[\int_{0}^{T} \int_{\mathcal{M}}\left(a(x) g^{2}\left(u_{t}\right)+a(x) u_{t}^{2}\right) d \mathcal{M} d t+\int_{0}^{T} \int_{\mathcal{M}}|u|^{2} d \mathcal{M} d t\right],
$$

for all $t \in(0, T)$, with $T$ large enough. The last inequality and (4.50) give us

$$
\bar{E}_{k}(t):=\frac{E_{k}(t)}{c_{k}^{2}} \leq \tilde{C}\left[\frac{\int_{0}^{T} \int_{\mathcal{M}}\left(a(x) g^{2}\left(u_{k}^{\prime}\right)+a(x) u_{k}^{\prime 2}\right)}{\int_{0}^{T} \int_{\mathcal{M}}\left|u_{k}\right|^{2} d \mathcal{M} d t}+1\right]
$$

From (4.40) and (4.51) we conclude that there exists a positive constant $\hat{M}$ such that

$$
\bar{E}_{k}(t):=\frac{E_{k}(t)}{c_{k}^{2}} \leq \hat{M}, \text { for all } t \in[0, T] \text { and for all } k \in \mathbb{N},
$$

that is,

$\frac{1}{2} \int_{\mathcal{M}}\left|\bar{u}_{k}^{\prime}\right|^{2} d \mathcal{M}+\frac{1}{2} \int_{\Omega}\left|\nabla \bar{u}_{k}\right|^{2} d \mathcal{M} \leq \hat{M}, \quad$ for all $t \in[0, T]$ and for all $k \in \mathbb{N}$.

For a subsequence $\left\{\bar{u}_{k}\right\}$, we obtain

$$
\begin{aligned}
& \bar{u}_{k} \rightarrow \bar{u} \text { weak star in } L^{\infty}(0, T ; V), \\
& \bar{u}_{k}^{\prime} \rightarrow \bar{u}^{\prime} \text { weak star in } L^{\infty}\left(0, T ; L^{2}(\mathcal{M})\right), \\
& \bar{u}_{k} \rightarrow \bar{u} \text { strongly in } L^{2}\left(0, T ; L^{2}(\mathcal{M})\right) .
\end{aligned}
$$

We observe that from (4.45) we deduce

$$
\begin{array}{r}
\lim _{k \rightarrow+\infty} \int_{0}^{T} \int_{\mathcal{M}} \frac{a(x) g^{2}\left(u_{k}^{\prime}\right)}{c_{k}^{2}} d \mathcal{M} d t=0 \\
\text { and } \lim _{k \rightarrow+\infty} \int_{0}^{T} \int_{\mathcal{M}} a(x)\left|\bar{u}_{k}^{\prime}\right|^{2} d \mathcal{M} d t=0 .
\end{array}
$$

In addition $\bar{u}_{k}$ satisfies the equation

$$
\bar{u}_{k}^{\prime \prime}-\Delta_{\mathcal{M}} \bar{u}_{k}+a(x) \frac{g\left(u_{k}^{\prime}\right)}{c_{k}}=0 \quad \text { on } \mathcal{M} \times(0, T) .
$$

Passing to the limit when $k \rightarrow+\infty$ taking the above convergence into account, we obtain

$$
\left\{\begin{aligned}
\bar{u}^{\prime \prime}-\Delta_{\mathcal{M}} \bar{u} & = & 0 & \text { on } \mathcal{M} \times(0, T) \\
\bar{u}^{\prime} & = & 0 & \text { on } \mathcal{M}_{*} \times(0, T)
\end{aligned}\right.
$$


Then, $v=\bar{u}_{t}$ verifies, in the distributional sense

$$
\left\{\begin{aligned}
v_{t t}-\Delta_{\mathcal{M}} v & =0 \text { on } \mathcal{M} \\
v & =0 \text { on } \mathcal{M}_{*}
\end{aligned}\right.
$$

Applying uniqueness results due to Triggiani and Yao [TRI-YAO] we obtain that $v=\bar{u}_{t}=0$. Returning to (4.57) we obtain, for a.e. $t \in(0, T)$ that

$$
\Delta_{\mathcal{M}} \bar{u}=0 \text { on } \mathcal{M}
$$

from what we deduce that $\bar{u}=0$, which is a contradiction in view of (4.49) and (4.55). The lemma is proved. $\square$

Inequalities (4.37) and (4.38) lead us to the following result.

Proposition 5.2.2: For $T>0$ large enough, the solution $\left[u, u_{t}\right]$ of (2.2) satisfies

$$
E(T) \leq C \int_{0}^{T} \int_{\mathcal{M}}\left[a(x)\left|u_{t}\right|^{2}+a(x)\left|g\left(u_{t}\right)\right|^{2}\right] d \mathcal{M} d t
$$

where the constant $C=C\left(T_{0}, E(0),\|a\|_{\infty}, a_{0}, \lambda_{1}, R,\|B\|, \frac{M}{\varepsilon^{2}}\right)$.

4.3. Conclusion of Theorem 3.1. In what follows we will proceed exactly as in Lasiecka and Tataru's work[LA-TA](see Lemma 3.2 and Lemma 3.3 of the referred paper) adapted to our context. Let

$$
\begin{aligned}
& \Sigma_{\alpha}=\left\{(t, x) \in \Sigma /\left|u_{t}\right|>1 \text { a. e. }\right\} \\
& \Sigma_{\beta}=\Sigma \backslash \Sigma_{\alpha} .
\end{aligned}
$$

Then using hypothesis (iii) in Assumption 2.1, we obtain

$$
\int_{\Sigma_{\alpha}} a(x)\left(\left[g\left(u_{t}\right)\right]^{2}+\left(u_{t}\right)^{2}\right) d \Sigma_{\alpha} \leq\left(k^{-1}+K\right) \int_{\Sigma_{\alpha}} a(x) g\left(u_{t}\right) u_{t} d \Sigma_{\alpha} .
$$

Moreover, from (3.1) and from the fact that $h\left(\frac{a(x)}{1+\|a\|_{\infty}} g\left(u_{t}\right) u_{t}\right) \leq h\left(a(x) g\left(u_{t}\right) u_{t}\right)$, we have

$$
\int_{\Sigma_{\beta}} a(x)\left(\left[g\left(u_{t}\right)\right]^{2}+\left(u_{t}\right)^{2}\right) d \Sigma_{\beta} \leq\left(1+\|a\|_{\infty}\right) \int_{\Sigma_{\beta}} h\left(a(x) g\left(u_{t}\right) u_{t}\right) d \Sigma_{\beta} .
$$

Then by Jensen's inequality

$$
\begin{aligned}
& \left(1+\|a\|_{\infty}\right) \int_{\Sigma_{\beta}} h\left(g\left(u_{t}\right) u_{t}\right) d \Sigma_{\beta} \\
\leq & \left(1+\|a\|_{\infty}\right) \text { meas }(\Sigma) h\left(\frac{1}{\text { meas }(\Sigma)} \int_{\Sigma} a(x) g\left(u_{t}\right) u_{t} d \Sigma\right) \\
= & \left(1+\|a\|_{\infty}\right) \text { meas }(\Sigma) r\left(\int_{\Sigma} a(x) g\left(u_{t}\right) u_{t} d \Sigma\right),
\end{aligned}
$$

where $r(s)=h\left(\frac{s}{\text { meas }(\Sigma)}\right)$ is defined in (3.2). Thus

$$
\begin{aligned}
\int_{\Sigma} a(x)\left(\left[g\left(u_{t}\right)\right]^{2}+\left(u_{t}\right)^{2}\right) d \Sigma \leq & \left(k^{-1}+K\right) \int_{\Sigma} a(x) g\left(u_{t}\right) u_{t} d \Sigma \\
& +\left(1+\|a\|_{\infty}\right) \text { meas }(\Sigma) r\left(\int_{\Sigma_{1}} a(x) g\left(u_{t}\right) u_{t} d \Sigma\right) .
\end{aligned}
$$


Splicing, together, (4.58) and (4.63), we have

$$
\begin{aligned}
E(T) \leq & \left(1+\|a\|_{\infty}\right) C\left[\frac{K_{0}}{\left(1+\|a\|_{\infty}\right)} \int_{\Sigma} a(x) g\left(u_{t}\right) u_{t} d \Sigma_{1}\right. \\
& \left.+ \text { meas }(\Sigma) r\left(\int_{\Sigma} a(x) g\left(u_{t}\right) u_{t} d \Sigma\right)\right]
\end{aligned}
$$

where $K_{0}=k^{-1}+K$. Setting

$$
\begin{aligned}
L & =\frac{1}{\operatorname{Cmeas}(\Sigma)\left(1+\|a\|_{\infty}\right)}, \\
c & =\frac{K_{0}}{\text { meas }(\Sigma)\left(1+\|a\|_{\infty}\right)},
\end{aligned}
$$

we obtain

$$
p[E(T)] \leq \int_{\Sigma} a(x) g\left(u_{t}\right) u_{t} d \Sigma=E(0)-E(T),
$$

where the function $p$ is as defined in (3.3). To finish the proof of Theorem 3.1, we invoke the following result from Lasiecka and Tataru [LA-TA]:

Lemma B. Let $p$ be a positive, increasing function such that $p(0)=0$. Since $p$ is increasing we can define an increasing function $q, q(x)=x-(I+p)^{-1}(x)$. Consider a sequence $s_{n}$ of positive numbers which satisfies

$$
s_{m+1}+p\left(s_{m+1}\right) \leq s_{m} .
$$

Then, $s_{m} \leq S(m)$, where $S(t)$ is a solution of the differential equation

$$
\frac{d}{d t} S(t)+q(S(t))=0, \quad S(0)=s_{0}
$$

Moreover, if $p(x)>0$ for $x>0$, then $\lim _{t \rightarrow \infty} S(t)=0$.

With this result in mind, we replace $T$ (resp. 0) in (4.64) with $m(T+1)$ (resp. $m T$ ) to obtain

$$
E(m(T+1))+p(E(m(T+1))) \leq E(m T), \text { for } m=0,1, \ldots
$$

Applying Lemma B with $s_{m}=E(m T)$ we deduce

$$
E(m T) \leq S(m), \quad m=0,1, \ldots
$$

Finally, using the dissipativity of $E(t)$ which is inherent in the relation (2.9), we have for $t=m T+\tau, 0 \leq \tau \leq T$,

$$
E(t) \leq E(m T) \leq S(m) \leq S\left(\frac{t-\tau}{T}\right) \leq S\left(\frac{t}{T}-1\right) \quad \text { for } t>T,
$$

where we have used above the fact that $S($.$) is dissipative. The proof of Theorem 3.1$ is now completed. 


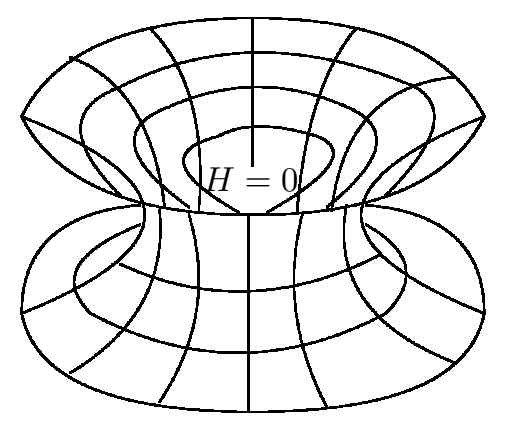

Fig. 2. Catenoid

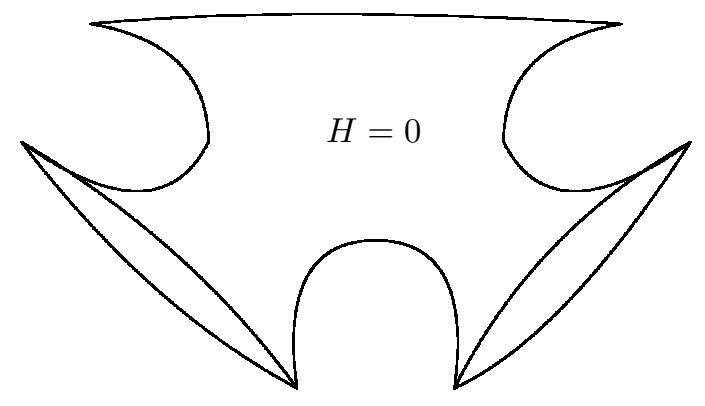

FIG. 3. Trinoid

4.4. Further Remarks. From the above procedure, we can construct a wide assortment of compact surfaces by jointing pieces of different kind of surfaces. However, according to the position of the observer (point $x_{0}$ ) the dissipative and the non dissipative areas can change drastically. To illustrate this, let us consider the Catenoid or the Trinoid (see figures 2 and 3 above) that are minimal surfaces, that is $H=0$.

Considering a strategic piece of one of these surfaces we can construct another compact surface according to the figure 4 below. Remember that the non dissipative regions must occur where $m(x) \cdot \nu(x) \leq 0, H \leq 0$ and simultaneously we are forced to consider $\left|k_{1}-k_{2}\right|$ sufficiently small (by parts). The dissipative area must contain strictly the closure of the points $x \in \mathcal{M}$ such that $m(x) \cdot \nu(x)>0$. It is not difficult to see that the non dissipative area in the figure $A$ can be located near the top and/or near $x_{0}$ while the non dissipative area in the figure $B$ can be located in the middle of surface and/or near $x_{0}$, assuming evidently that $k_{1} \approx k_{2}$ on these non dissipative areas. 
Fig. $A$

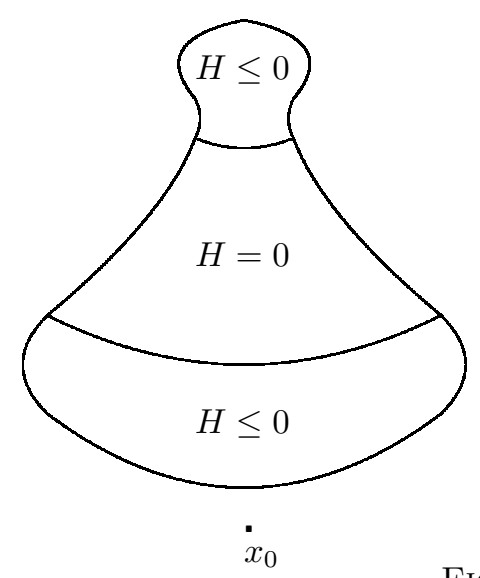

FIG. 4.

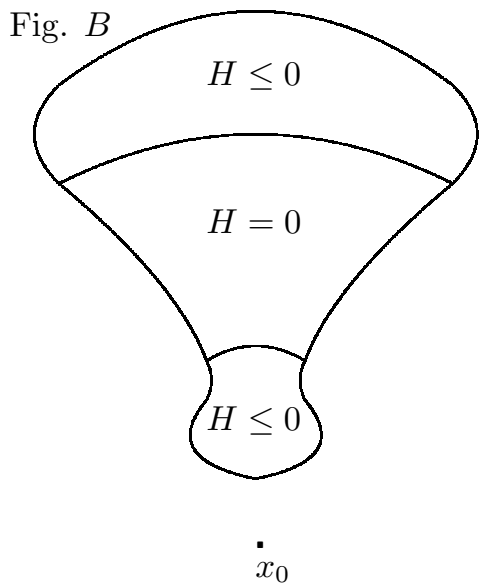

REFERENCES

$[\mathrm{ALA}]$

F. Alabau-Boussouira, Convexity and weighted integral inequalities for energy decay rates of nonlinear dissipative hyperbolic systems, Appl. Math. Optim., 51:1 (2005), pp. 61-105.

[BAR] C. Bardos, G. Lebeau AND J. Rauch, Control and stabilisation de l'equation des ondes, Appendix II in J. L. Lions controllabilité exacte des systèmes distribués, Collection RMA, Vol. 8, Masson, Paris, 1988.

$[\mathrm{LOH}]$ R. BEY, A. HEMINNA AND J.P. LOHÉAC, Boundary stabilization of the linear elastodinamic system by a Lyapunov-type method, Revista Matemática Complutense, 16:2 (2003), pp. 417-441.

[CA-DO] M.M. Cavalcanti and V.N. Domingos Cavalcanti, Existence and asymptotic stability for evolution problems on manifolds with damping and source terms, J. Math. Anal. Appl., 291:1 (2004), pp. 109-127.

[CAVAL] M.M. CAVAlCANTi AND H.P. OQUendo, Frictional versus viscoelastic damping in a semilinear wave equation, SIAM J. Control Optim., 42:4 (2003), pp. 13101324.

[An1] D. Andrade, M.M. Cavalcanti, V.N. Domingos Cavalcanti and H.P. OQuendo, Existence and asymptotic stability for viscoelastic evolution problems on compact manifolds, J. Comput. Anal. Appl, 8:3 (2006), pp. 173-193.

[An2] D. Andrade, M.M. Cavalcanti, V.N. Domingos Cavalcanti and H.P. OQUeNDO, Existence and asymptotic stability for viscoelastic evolution problems on compact manifolds (part II), J. Comput. Anal. Appl, 8:3 (2006), pp. 287-301.

[CA-DO-LA] M.M. Cavalcanti, V.N. Domingos Cavalcanti and I. Lasiecka, Wellposedness and optimal decay rates for wave equation with nonlinear boundary dampingsource interaction, Journal of Differential Equations, 236 (2007), pp. 407-459.

[CHR] H. Christianson, Semiclassical non-concentration near hyperbolic orbits, J. Funct. Anal., 246:2 (2007), pp. 145-195.

[DOCARMO] M. Do CARmo, Differential Geometry of Curves and Surfaces, Prentice Hall, New Jersey, 1976.

[HEM1] A. Heminna, Stabilization frontière de problèmes de Ventcel (Boundary stabilization of Venttsel problems), ESAIM, Control Optim. Calc. Var., 5 (2000), pp. 591-622.

[HEM2] A. HEMINNA, Exact controllability of the linear elasticity system with evolutive Ventcel conditions, Port. Math., 58:3 (2001), pp. 271-315. 
[HEM3]

[HIT]

[LEM1]

[LEM2]

[LEM3]

[LA-TA]

[LA-TRI]

[LIONS1]

[LIONS2]

[LiMa]

[Liu]

[Mar]

[Na1]

[Na2]

[NE]

[Ra-Ta]

[TRI-YAO]

[Tou]

A. Heminna, Contrôlabilité exacte d'un problème avec conditions de Ventcel évolutives pour le système linéaire de l'élasticité (Exact controllability of a problem with evolutive Venttsel conditions for the linear elasticity system), Rev. Mat. Complut., 14:1 (2001), pp. 231-270.

M. HitRIK, Expansions and eigenfrequencies for damped wave equations, Journées "Équations aux Dérivées Partielles" (Plestin-les-Grèves, 2001), Exp. No. VI, 10 pp., Univ. Nantes, Nantes, 2001.

A. Khemmoudj and M. Medjden, Exponential Decay for the Semilinear Damped Cauchy-Ventcel Problem, Bol. Soc. Paran. Mat., 22:2 (2004), pp. 97-116.

K. Lemrabet, Problème aux limites de Ventcel dans un domaine non régulier, CRAS Paris, t.300, Série I, no. 15 (1985), pp. 531-534.

K. LEMrABet, Etude de divers problèmes aux limites de Ventcel d'origine physique ou mécanique dans des domaines non réguliers, Thèse, USTHB, Alger, (1987).

K. Lemrabet And D.E. Teniou, Controlabilité exacte pour l'equation des ondes avec conditions aux limites de type Cauchy-Ventcel, Publication de l'institut de Mathématique, U.S.T.H.B.

I. LASIECKA AND D. TATARU, Uniform boundary stabilization of semilinear wave equation with nonlinear boundary damping, Differential and integral Equations, 6 (1993), pp. 507-533.

I. LASIECKA AND R. TRIGGIANI, Uniform stabilization of the wave equation with Derichlet or Neumann feedback control without geometric conditions, Appli. Math. Optim., 25 (1992), pp. 189-124

J.L. Lions, Controlabilité exacte, perturbations et stabilisation de systèmes distribués, tome 1, Masson, 1988.

J.L. Lions, Controlabilité exacte, perturbations et stabilisation de systèmes distribués, tome 2, Masson, 1988.

J.L. Lions and E. Magenes, Problèmes Aux Limites Non Homogènes et Applications, Dunod, Paris, 1968, Vol. 1.

K. LIU, Localy distributed control and damping for conservative systems, SIAM J. Control and Optimization, 35:5 (1997), pp. 1574-1590.

P. Martinez, A new method to obtain decay rate estimates for dissipative systems with localized damping, Rev. Mat. Complutense, 12:1 (1999), pp. 251-283.

M. NAKAO, Decay of solutions of the wave equation with a local nonlinear dissipation, Math. Ann., 305 (1996), pp. 403-417.

M. NAKAO, Decay of solutions of the wave equation with local degenerate dissipation, Israel J. of Maths., 95 (1996), pp. 25-42.

J.C. Nedelec, Ondes acoustiques et électromagnétiques, Équations intégrales, Cours de DEA, Ecole polytechnique, Palaiseau. France 1996.

J. RAUCH AND M. TAYLOR, Decay of solutions to $n$ ondissipative hyperbolic systems on compact manifolds, Comm. Pure Appl. Math., 28:4 (1975), pp. 501-523.

R. Triggiani and P.F. YAO, Carleman estimates with no lower-Order terms for general Riemannian wave equations, Global uniqueness and observability in one shot, Appl. Math. and Optim, 46 (Sept./Dec. 2002), pp. 331-375. Special issue dedicated to J. L. Lions.

D. ToundyKov, Optimal decay rates for solutions of nonlinear wave equation with localized nonlinear dissipation of unrestricted growth and critical exponents source terms under mixed boundary, Nonlinear Analysis T. M. A. (to appear).

E. ZUAZUA, Exponential decay for the semilinear wave equation with locally distributed damping, Commun. Partial Differential Equations, 15:2 (1990), pp. 205235 . 
M. M. CAVALCANTI ET AL. 